\title{
Acute retropharyngeal abscess in adults: a case series
}

\section{Inderdeep Singh*, Vikas Gupta, Sunil Goyal, Manoj Kumar, Lakshmi Ranjit, Salil Gupta}

Department of ENT, Command Hospital, Pune, India

Received: 08 November 2016

Accepted: 28 November 2016

\author{
*Correspondence: \\ Dr. Inderdeep Singh, \\ E-mail: dridsingh@hotmail.com
}

Copyright: (c) the author(s), publisher and licensee Medip Academy. This is an open-access article distributed under the terms of the Creative Commons Attribution Non-Commercial License, which permits unrestricted non-commercial use, distribution, and reproduction in any medium, provided the original work is properly cited.

\begin{abstract}
Retropharyngeal abscesses are uncommon but potentially lethal infections. In the adult group they are usually secondary to trauma, foreign bodies, or as a complication of dental infections. Early diagnosis and the wide spread use of antibiotics have made these infections less common today. High index of suspicion and clinical acumen is required to provisionally diagnose these cases. In the current era where empirical use of high end and advanced antibiotics is prevalent it is not surprising to find patients with highly resistant microbes, such cases present further difficulty to treat. For large retropharyngeal abscesses, surgical drainage remains the primary modality followed by aggressive targeted antibiotic therapy. We present three cases of deep neck space infections with primary focus in retropharyngeal space. All required surgical drainage and aggressive postoperative care. Extensive review of literature of retropharyngeal abscess in adults was done to derive characterizations about this disease.
\end{abstract}

Keywords: Retropharyngeal abscess, Deep neck space infection, Retropharyngeal space

\section{INTRODUCTION}

Acute retropharyngeal abscesses are generally described as a disease of children. Acute retropharyngeal abscesses in adults are an uncommon but potentially lethal infection involving deep neck spaces. It is usually secondary to trauma, foreign bodies, or as a complication of dental infections. ${ }^{1}$

It was more common in the preantibiotic era and more than $95 \%$ of the cases involved children less than 6 years. ${ }^{2}$ However, review of existing literature reveals a paucity of information on the subject in adults. The clinical presentation and preceding medical history may differ in various age groups with retropharyngeal abscess.

In this article we present a review of 3 consecutive cases of acute onset deep neck abscesses with involvement of the retropharyngeal space. All the 3 cases were successfully managed by securing of the airway early and urgent drainage of the abscess by cervical approach along with broad spectrum injectable antibiotics.

\section{CASE REPORT}

\section{Case 1}

A 46 years old male presented to emergency department of our hospital with complaints of sore throat of 10 days duration, progressive dysphagia and odynophagia of 6 days duration and progressive neck swelling for last 5 days. There was no history of difficulty in breathing or noisy breathing, dyspnoea, trismus, previous trauma/ foreign body ingestion or dental problems. Patient had no known comorbidity.

On examination patient was afebrile with normal vital parameters. Otorhinolaryngology evaluation revealed a diffuse neck swelling involving both sides of neck and extending superiorly from level of mandible to inferiorly up to clavicle. Posteriorly it extended up to trapezius which was more on left side then right side as shown in Figure 1. Swelling was tense and skin over the swelling was warm, erythematous and indurated. Examination of oral cavity and oropharynx revealed satisfactory dental 
hygiene with adequate mouth opening. Bulging of posterior pharyngeal wall could be seen. Hopkins rod examination revealed a normal glottis and adequate glottic chink.

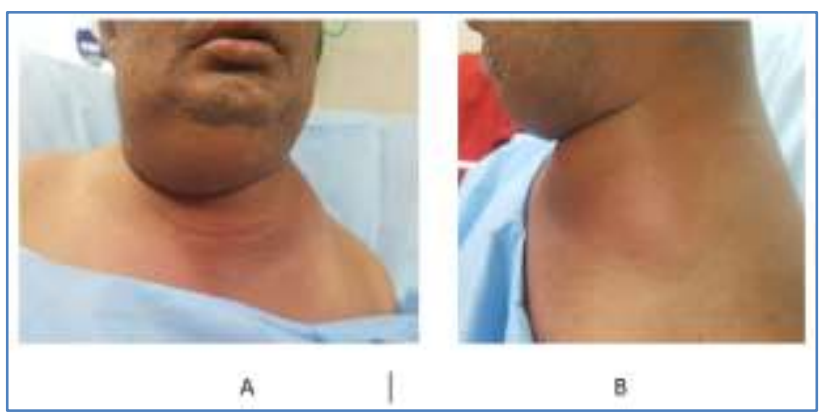

Figure 1: A \& B showing the extent of neck swelling at initial presentation to the emergency department of the hospital.

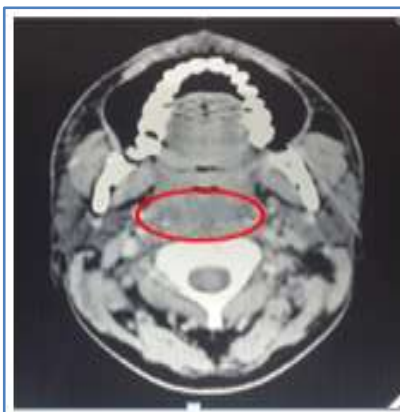

A

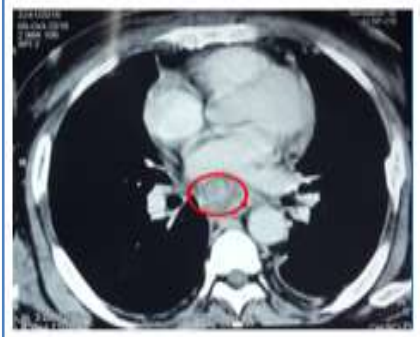

C

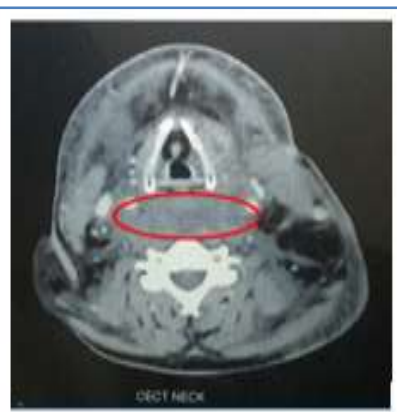

B

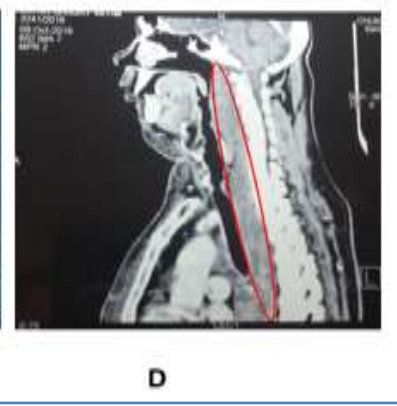

Figure 2: A \& B: Axial cuts of suprahyoid and infrahyoid region showing collection in the retropharyngeal and retrovisceral spaces respectively.

$C$ : Axial cut showing collection in the posterior mediastinum with indentation on the left atrium. D: Sagittal cut showing the collection in danger space extending from the skull base up to the diaphragm with involvement of the posterior mediastinum.

An ultrasound of the neck done 2 days prior showed had only shown subcutaneous edema and features suggestive of cellulitis. The patient was started on oral antibiotics by his primary physician. As the airway was stable at presentation, an urgent CECT of neck and chest was asked for which revealed an ill-defined necrotic peripherally enhancing collection epicenterd in the retropharyngeal space extending to prevertebral and danger spaces posteriorly, laterally to bilateral parapharyngeal spaces with involvement of carotid space and displacment internal jugular vein laterally. There was also involvement of the anterior visceral, strap muscle on the left side and thrombosis of the left internal jugular vein. Inferiorly it extended into the posterior mediastinum with indentation of the left atrium and anterior displacement of the cardiac structures as shown in Figure 2.

Patient was started on broad spectrum injectable antibiotics and planned to be taken up for an urgent incision and drainage through the cervical approach under general anaesthesia. An awake fibreoptic guided nasal intubation was performed initially to secure the airway. A horizontal skin incision was given at the level of cricoid extending from the anterior border of one sternocleidomastoid to the other. Surgical steps are depicted in Figure 3. Intraoperatively dark green to black pus was drained from bilateral parapharyngeal spaces, retropharyngeal and anterior visceral space. Left IJV was found to be thrombosed. A tracheostomy was done through a separate incision so as to maintain the airway till the edema and pus from the neck subsides.

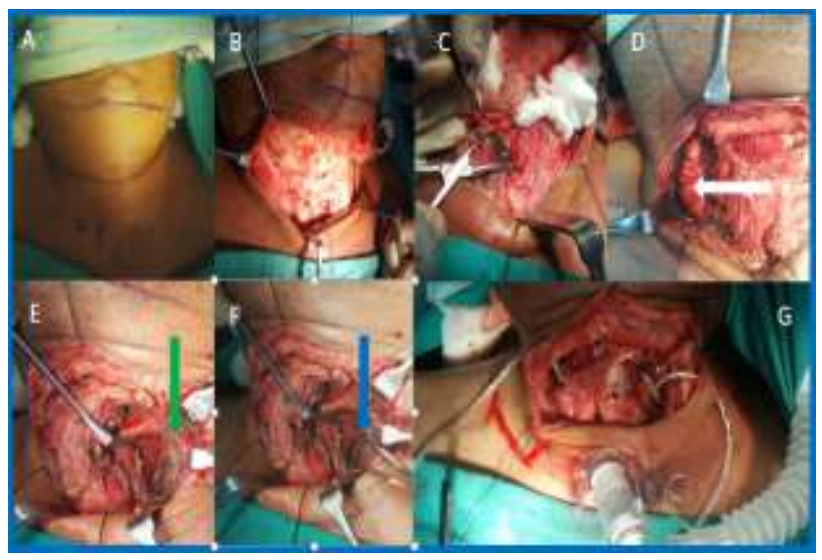

Figure 3: A: Showing the planning of the cervical incision. B: Elevation of skin flaps. C: Opening of the anterior visceral space with pus pockets (dark green colour pus). D: Showing pus in right parapharyngeal space with lateral displacement of internal jugular vein (White arrow). E: Green arrow pointing towards thrombosed left internal jugular vein. F: Blue arrow pointing towards retropharyngeal space. G: Showing 2 drains in situ secured with temporary tracheostomy through a separate incision.

Pus sent for culture was positive for E. coli and the antibiotics were changed based on the sensitivity report. Patient responded well to the surgical intervention along with injectable antibiotics. Patient was decannulated after 2 weeks and at the same time the drain was also removed as shown in Figure 4. The staples were removed the next day following which the patient was discharged. He was kept on follow up till the closure of the tracheostoma. 


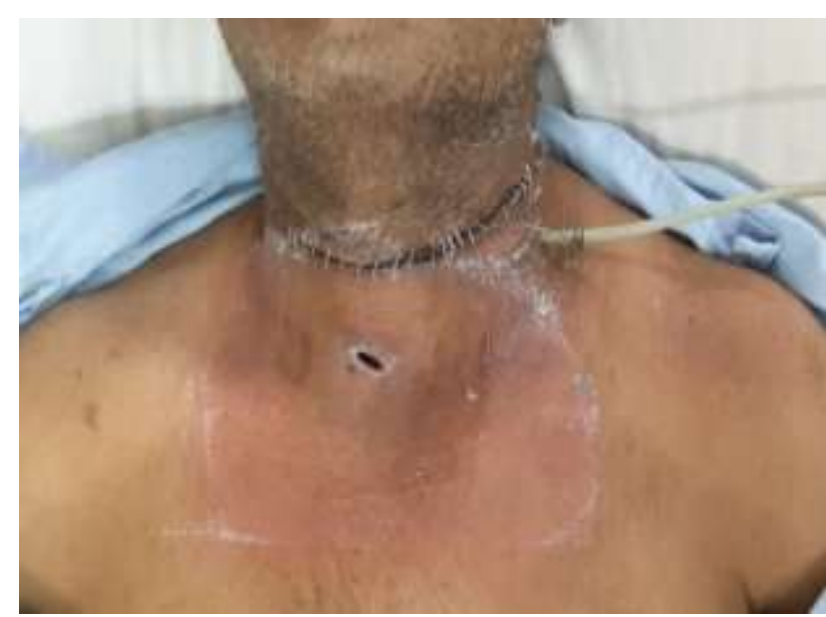

Figure 4: Showing the surgical site with drain in situ on left side and healing tracheostoma. The neck swelling has completely resolved.

\section{Case 2}

A 72 years old female was referred to our hospital (with endotracheal tube intubation) with complaints of progressive odynophagia, dysphagia and diffuse neck swelling since 5 days, and trismus for the past 2 days. There was no history of noisy breathing or dyspnoea. She gave history of a fish bone ingestion 5 days back prior to onset of the symptoms. Patient had no known comorbidity.

On examination patient was afebrile with normal vital parameters. Otorhinolaryngology evaluation revealed diffuse (R) sided neck swelling from hyoid to clavicle. Examination of oral cavity and oropharynx revealed trismus with mouth opening limited to $2 \mathrm{~cm}$. Hopkins rod examination revealed a reduced glottis chink due to edema of aretenoids and epiglottis.

An urgent CECT neck and chest was requested which revealed heterogeneously enhancing collection extending from skull base to the level of C6 vertebral body involving the retropharyngeal space and extending into the right parapharyngeal space and tracking along the carotid sheath and right lobe of thyroid medially as shown in Figure 5.

Patient was started on broad spectrum injectable antibiotics and an urgent incision and drainage of the abscess was done through the cervical approach under general anaesthesia. Tracheostomy was performed at the end of the surgical intervention for securing the airway. Intraoperatively dark yellowish pus was drained from the retropharyngeal, right parapharyngeal and carotid spaces.

Aggressive broad spectrum antibiotics were continued in the postoperative period as the culture report did not show any growth. Drain was removed after 7 days postoperatively. Patient was decannulated and discharged on $14^{\text {th }}$ postoperative day.

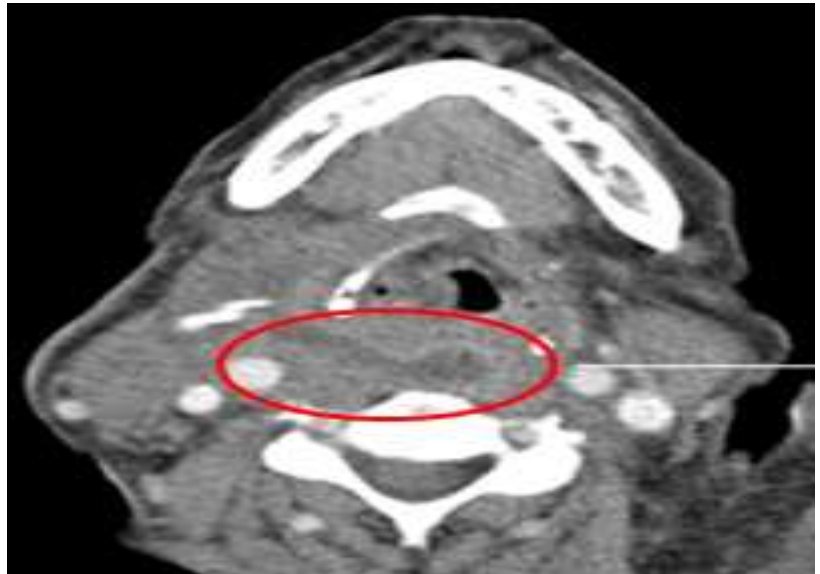

Figure 5: Showing collection of pus in retropharyngeal space and right parapharyngeal spaces.

Case 3

A 74 year old male presented with progressive odynophagia and dysphagia and progressive neck swelling for 5 days and restricted mouth opening for 2 days. There was no history of dyspnea or respiratory distress. Patient did not give any history of dental problems or history of trauma or foreign body ingestion. Patient had no known comorbidity.

On examination patient was afebrile with normal vital parameters. An otolaryngological examination revealed swelling in midline of neck extending from the mandible to the clavicle as shown in Figure 6. Examination of oral cavity and oropharynx revealed trismus with mouth opening limited to $1 \mathrm{~cm}$. Rigid Hopkins examination could not be performed because of the severe trismus.

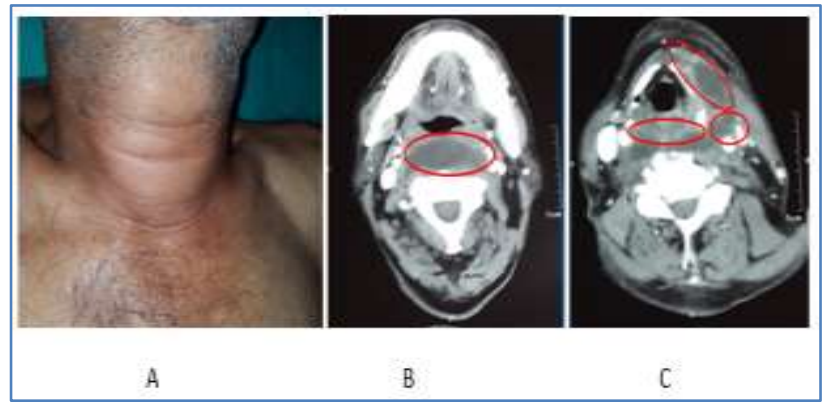

Figure 6: A: Showing midline neck swelling in a patient with retropharyngeal abscess. B: Axial CT scan showing involvement of retropharyngeal space in suprahyoid neck. C: Axial CT scan showing involvement of retrovisceral space, left parapharyngeal space, muscular space and anterior visceral space in infrahyoid neck.

An urgent CT scan of neck and chest was requested which revealed heterogeneously enhancing collection involving the retropharyngeal space extending from skull 
base to superior mediastinum. There was evidence of collection in the left parapharyngeal space, muscular space and anterior visceral space. Figure 6 (B \& C) shows the CT scan of the patient.

Patient was started on broad spectrum injectable antibiotics and an urgent incision and drainage of the abscess was done through the cervical approach along with tracheostomy under general anaesthesia. Intraoperatively pus was drained from the retropharyngeal, left parapharyngeal spaces, muscular space and anterior visceral space.

Pus culture was positive was Staphlococcus aureus and antibiotics was changed to culture directed sensitivity report. Drain was removed after 7 days post operatively. Patient was decannulated and discharged on $14^{\text {th }}$ postoperative day.

\section{DISCUSSION}

The retropharyngeal space is a potential space which lies between the visceral (buccopharyngeal) fascia covering the posterior pharynx and esophagus and the alar fascia (a division of the deep layer of deep cervical fascia). ${ }^{3}$ It extends from the base of the skull down into the superior mediastinum, where the visceral and alar fascia fuses at the level of the second thoracic vertebra. Laterally, it is bounded by the carotid sheath.

Acute retropharyngeal abscess is usually unilateral and primarily seen early in childhood because these lymph nodes tend to regress with age. ${ }^{3}$ Upper respiratory infections cause most retropharyngeal disease in children because these lymph nodes receive drainage from the nose, sinuses, and pharynx. ${ }^{3}$ Acute retropharyngeal abscess may be seen both in children and adults following trauma to the posterior pharynx with secondary infection. ${ }^{1,3}$ In our series of adults patients with acute retropharyngeal abscess 2 of the cases had a preceding history of upper respiratory tract infection while one of the patient had a history suggestive of trauma to the pharynx due to fish bone ingestion. However, other less common etiological factors such as tuberculosis, syphilis and vertebral fractures must be ruled out with appropriate clinical evaluation and investigations.

de Clercq LD et al in its paper on retropharyngeal abscess in adults have stated that patients usually present with fever, odynophagia, dysphagia, dyspnoea, drooling, cervical rigidity (torticollis), a 'hot potato' or hyponasal voice, and sepsis. ${ }^{4}$ While on inspection one might see a bulging of the pharyngeal wall. The mucosa itself may be swollen and inflamed. ${ }^{1,4}$ The most common presentation in our series of patients was acute onset progressive odynophagia, dysphagia and neck swelling suggesting an underlying acute inflammatory condition. However, none of our patient had any features of sepsis at presentation.
The causative organism can vary depending on the source of infection. Most cases are polymicrobial and involve both aerobes and anaerobes. ${ }^{3,5,6}$ In our case series one of the patient cultured for $E$ coli while another culture positive for Staphloccoccus aureus. Culture report guides the choice of antibiotics. However, in the absence of a positive culture report the management should include aggressive broad spectrum injectable antibiotics.

CECT of the head and neck is the radiological investigation of choice to confirm the diagnosis and evaluate for spread of infection into adjacent deep neck spaces. ${ }^{3,7}$ This was the primary modality of investigation in all our cases. Other radiological investigations include plain radiograph, ultrasonography (USG) and magnetic resonance imaging. USG is especially useful because of its easy availability, bedside procedure, guided aspiration of pus, avoids radiation exposure and repeat follow up imaging.

The most worrisome complications of acute retropharyngeal infection are airway compromise and rupture of an abscess leading to aspiration pneumonia. Patients who have signs of airway compromise should be taken immediately to the operating room for securing the airway. One of our patients had a compromised airway at presentation and was referred to us with an endotracheal tube in situ. Fiber optic nasotracheal intubation under local anesthesiais especially useful in patients who have severetrismus but whose airways are otherwise uncompromised..$^{3,8}$ In one of the patient with trismus and post pharyngeal bulge and neck stiffness (case 1) we initially secured the airway with a fibreoptic guided nasotracheal intubation. All our cases were converted to tracheostomy after drainage of the abscess via cervical approach.

Surgical intervention is the mainstay of treatment for more complicated or severe cases of deep neck space infection. Indications for surgery include airway compromise, critical condition, septicemia, complications, descending infection, diabetes mellitus, or no clinical improvement within 48 hours of the initiation of parenteral antibiotics. ${ }^{9}$ Abscesses greater than $3 \mathrm{~cm}$ in diameter that involve the prevertebral, anterior visceral, or carotid spaces, or that involve more than two spaces, should be surgically drained. ${ }^{3,9}$ Surgical drainage can be performed in several ways, including simple intraoral or extraoral incision and drainage for superficial abscesses, a more extensive external cervical approach for deeper and more complicated infections. ${ }^{3}$ Large and multilocular abscesses usually require incision and drainage. ${ }^{3,5}$ In our reported case series all the abscesses were urgently drained via cervical approach under general anesthesia because retropharngeal abscess was associated with multiple deep neck space involvement and complications. Wide exposure of the infected tissue is important, as is debridement of any necrotic tissue that is present. Wounds with less necrosis can be closed at surgery with active suction drainage. ${ }^{3}$ 
Lemierre syndrome is the suppurative thrombophlebitis of the internal jugular vein, as a result of extension of infection into carotid space. Pathognomonic findings include swelling and tenderness at the angle of the jawand along the sternocleidomastoid muscle, along with signs of sepsis (spiking fevers, chills) and evidence of pulmonary emboli. ${ }^{10,11}$ One of our patient (Case 1) had thrombosis of the internal jugular vein which was confirmed both by radiology and intraoperative finding.

Mediastinitis can result from downward extension of deep neck space infection and can have lethal outcomes. ${ }^{12}$ Urgent transcervical drainage of the abscess can achieve satisfactory drainage of abscess from the superior mediastinum. In the event of inefficacy of transcervical drainage, more aggressive transthoracic drainage (sternotomy or thyrotomy) of abscess may be required. ${ }^{12}$ In our case series none of the patient required a transthoracic drainage of the abscess.

\section{CONCLUSION}

The aim of discussion this case series was to highlight the mode of presentation of a retropharyngeal abscess. Successful outcomes depend on early identification and urgent aggressive management. The main highlight of the management should be to secure the airway along with evacuation of the pus by surgical drainage and aggressive intravenous antibiotic therapy which should preferable is culture directed.

Funding: No funding sources

Conflict of interest: None declared

Ethical approval: Not required

\section{REFERENCES}

1. Goldenberg D, Golz A, Joachims H. Retropharyngeal abscess: a clinical review. J Laryngol Otol. 1997;111(6):546-50.

2. Seid AB, Dunbar JS, Cotton RT. Retropharyngeal abscesses in children revisited. Laryngoscope. 1979;89(11):1717-24.

3. Vieira F, Allen SM, Stocks RMS, Thompson JW. Deep neck infection. Otolaryngol Clin North Am. 2008;41(3):459-83.
4. de Clercq LD, Chole RA. Retropharyngeal abscess in the adult. Otolaryngol Head Neck Surg. 1980;88(6):684-9.

5. Tan P-T, Chang L-Y, Huang Y-C, Chiù C-H, Wang $\mathrm{C}-\mathrm{R}$, Lin T-Y. Deep neck infections in children. Journal of microbiology, immunology, and infection. Wei Mianyugan Ran Zazhi. 2001;34(4):287-92.

6. Lee J-K, Kim H-D, Lim S-C. Predisposing factors of complicated deep neck infection: an analysis of 158 cases. Yonsei Med J. 2007;48(1):55-62.

7. Freling N, Roele E, Schaefer Prokop C, Fokkens W. Prediction of deep neck abscesses by contrast enhanced computerized tomography in 76 clinically suspect consecutive patients. Laryngoscope. 2009;119(9):1745-52.

8. Gidley PW, Ghorayeb BY, Stiernberg CM. Contemporary management of deep neck space infections. Otolaryngol Head Neck Surg. 1997;116(1):16-22.

9. Boscolo-Rizzo P, Marchiori C, Zanetti F, Vaglia A, Da Mosto MC. Conservative management of deep neck abscesses in adults: the importance of CECT findings. Otolaryngol Head Neck Surg. 2006;135(6):894-9.

10. Brook I. Microbiology and management of deep facial infections and Lemierre syndrome. ORL J Otorhinolaryngol Relat Spec. 2003;65(2):117-20.

11. Brook I. Microbiology and management of peritonsillar, retropharyngeal, and parapharyngeal abscesses. J Oral Maxillofac Surg. 2004;62(12):1545-50.

12. Kovacić M, Kovacić I, Dželalija B. Descending necrotizing mediastinitis secondary to retropharyngeal abscess. Acta Clin Croat. 2015;54(4):541-6.

Cite this article as: Singh I, Gupta V, Goyal S, Kumar M, Ranjit L, Gupta S. Acute retropharyngeal abscess in adults: a case series. Int J Otorhinolaryngol Head Neck Surg 2017;3:151-5. 